Pacific Journal of Mathematics

ON RINGS ON GROUPS

March 1974 


\title{
ON RINGS ON GROUPS
}

\author{
J. D. REID
}

The class of faithful (fully faithful) abelian groups is introduced as a generalization of the semi-simple (strongly semi-simple) groups recently discussed by $\mathbf{R}$. A. Beaumont and D. A. Lawver. A group is faithful if it admits some associative ring structure with trivial left annihilator. Fully faithful groups are the nonnil groups such that every nontrivial associative ring structure has trivial left annihilator. Several of the results of Beaumont and Lawver are generalized and it is shown that fully faithful groups arise naturally in classifying strongly indecomposable torsion free groups according to the ring structures they support.

Several authors have considered the question of what sorts of ring structures a given additive abelian group will support. The comments which follow were inspired by a recent paper along these lines of Beaumont and Lawver [3]. Our aim is to obtain the results of [3] in as self-contained a way as possible. We do this by focusing on the relation between a group and its endomorphism ring and, as it turns out, we can base our whole discussion-almost-on certain general facts and ideas taken from [5]. Aside from the fact that we obtain in one instance (Theorem 1) a more general result, with a shorter proof, than in [3], and in another instance (Theorem 5) more information than in [3], we hope that the adoption of a single point view provides better insight into what is involved. We also include a few results not found in [3]. In the last few theorems our resolve crumbles somewhat and we admit one additional piece of evidence-the Beaumont-Pierce analogue of the Wedderburn Principal Theorem (cf. [1]).

Finally, we would like to thank the authors of [3] for a preprint of their paper.

If $R$ is a ring with additive group $G$ we have available the regular representation of $R$-i.e., the map $\phi$ of $R$ into the endomorphism ring $E=E(G)$ of $G$ such that, for $x, y \in R, \varphi(x) y=x y$. This map $\varphi$ is a ring homomorphism of $R$ into $E$, but we may describe it in other terms. The associative law gives

$$
\varphi(\varphi(x) y)=\varphi(x) \varphi(y) \quad x, y \in G .
$$

This equation carries with it the information that $\varphi(G)$ is closed under the multiplication in $E$. Conversely given $\varphi \in \operatorname{Hom}_{Z}(G, E)$ satisfying 
(1), we may define $x \cdot y=\varphi(x) y$ for $x, y \in G$ and obtain an associative ring with additive group $G$ and left regular representation $\varphi$. Thus motivated, we make the

Definition. A ring on the abelian group $G$ is an element $\varphi \in \operatorname{Hom}_{z}(G, E)$ such that $\varphi(\varphi(x) y)=\varphi(x) \varphi(y)$ for all $x, y \in G$.

Given any subring $A$ of $E=E(G)$, an element $\varphi \in \operatorname{Hom}_{A}(G, A)$ is always a ring on $G$. Conversely, if $\varphi$ is a ring on $G$ then $\varphi(G)$ is a subring of $E$ and $\varphi \in \operatorname{Hom}_{\varphi(G)}(G, \varphi(G))$.

It is easy to show that two rings $\varphi, \varphi^{\prime}$ on $G$ give isomorphic ring structures if and only if $\varphi^{\prime}(x)=\beta^{-1} \varphi(\beta x) \beta, x \in G$ holds for some unit $\beta \in E$. This, and most of the above, has been observed in Fuchs [4].

Notation. Write $(G, \varphi)$ for the associative ring given by the ring $\varphi$ on $G$.

CoRollary 0. If the left annihilator of $G$ in $(G, \varphi)$ is trivial, then $\varphi$ is $1-1$.

Proof. $K=\operatorname{ker} \varphi=$ left annihilator of $G$ in $(G, \varphi)$.

Special cases. (i) $(G, \varphi)$ has no nilpotent ideals $\Rightarrow \varphi$ is $1-1$. (Proof. $K^{2}=0$. .)

(ii) $(G, \varphi)$ semi-simple $\Rightarrow \varphi$ is $1-1$. (Proof. Radical $\supseteqq$ all nilpotent ideals.)

This suggests our basic distinction.

Definition. An abelian group is faithful if there is some ring $\varphi$ on $G$ with $\operatorname{ker} \varphi=0$. An abelian group $G$ is fully faithful if $G$ is nonnil (i.e., $G$ admits some nonzero associative ring structure) and every ring $\varphi$ on $G$ has $\operatorname{ker} \varphi=0$.

Beaumont and Lawver call a group $G$ semi-simple (strongly semisimple) if $G$ has some semi-simple ring structure (respectively if $G$ is nonnil and every nonzero ring on $G$ is semi-simple). Clearly, faithful groups are nonnil, semi-simple groups are faithful and strongly semi-simple groups are fully faithful. The following theorem (essentially) generalizes Theorem 4.2 of [3]. But first we need a

Lemma. Let $R$ be a ring with a trivial left annihilator: 


$$
l(R)=\{a \in R \mid a R=0\}=0 .
$$

Suppose $A$ and $B$ are subsets of $R$ such that

$$
\begin{gathered}
R=A+B=\{a+b \mid a \in A, b \in B\}, \\
A^{2}=\left\{a a^{\prime} \mid a, a^{\prime} \in A\right\}=0 \text { and } B^{2}=\left\{b b^{\prime} \mid b, b^{\prime} \in B\right\}=0 .
\end{gathered}
$$

Then $R=0$.

Proof. Our hypotheses yield $A \subseteq l(A), B \subseteq l(B)$, and $l(A) \cap l(B) \subseteq$ $l(R)=0$. Since $R=A+B$ we now have $R=l(A) \oplus l(B)$. Clearly now, $l(A)=A$ and $l(B)=B$. Since $l(A)$ is a left ideal, $A$ is a left ideal. But then $l(A)$ is a 2 -sided ideal, so $A$ is too. Similarly $B$ is a 2-sided ideal. We now have $R=A \oplus B, A^{2}=B^{2}=0, A$ and $B$ 2-sided ideals of $R$, so $R^{2}=0$. Thus $R \subseteq l(R)=0$ as required.

Theorem 1. A fully faithful group is indecomposable.

Proof. Let $G$ be fully faithful and let $\varphi$ be a nonzero ring on $G$. Suppose $G=A \oplus B$ with projections $\alpha: G \rightarrow A$ and $\beta=1-\alpha$. By the lemma, we cannot have both $A^{2}=0$ and $B^{2}=0$, without $G=0$ in which case we agree that $G$ is indecomposable. Thus either both $A$ and $B$ are subrings of $G$ with one, say $A$, satisfying $A^{2} \neq 0$; or one of $A, B$, say $A$, is not a subring of $G$.

In the former case (i.e., $\varphi(a) a^{\prime} \in A$ for all $a, a^{\prime} \in A$ ), for each $a \in A, \alpha \varphi(a) \alpha=\varphi(a) \alpha$ holds in $E$. Using this it follows that the definition

$$
\psi(x)=\varphi(\alpha x) \alpha
$$

yields a nonzero ring on $G$ with $B \subseteq \operatorname{ker} \psi$.

In the latter case (and here we follow Beaumont-Lawver), since $\varphi(a) a^{\prime} \in A$ for some pair $a, a^{\prime} \in A$, the map $\psi: G \rightarrow E$ given by

$$
\psi(x)=\beta \varphi(\alpha x) \alpha
$$

is nonzero and satisfies $\psi(x) G \leqq B, \psi(x) B=0$ for all $x \in G$. Thus $\psi(\psi(x) y)=0=\psi(x) \psi(y)$ for all $x, y \in G$ so that (1) holds for $\psi$.

Thus in any case a nontrivial decomposition of $G$ leads to a nonnil nonfaithful ring on $G$, proving the theorem.

COROLLARY 1.1. An abelian group is torsion and fully faithful if and only if $G=Z(p)$ for some prime $p$. Fully faithful groups cannot be mixed. 
Proof. By the above, a torsion fully faithful group $G$ has the form $Z\left(p^{k}\right)$ for some $k, 1 \leqq k \leqq \infty$. If $1<k<\infty$, the obvious ring on $Z\left(p^{k}\right)$ can be modified to $x \cdot y=(p x) y$, giving a ring on $Z\left(p^{k}\right)$ with nontrivial left annihilator; if $k=\infty, \operatorname{Hom}\left(Z\left(p^{\infty}\right), E\right)=0$. Hence indeed $G=Z(p)$. The converse is trivial. The last statement comes from the well known fact that there are no indecomposable mixed groups.

Corollary 1.2. (cf. [3], Theorems 2.1, 2.2, Corollary 2.3). An abelian group $G$ is torsion and strongly semi-simple if and only if $G=Z(p)$ for some prime $p$. Every mixed group admits a ring structure with nonzero nilpotent ideals.

\section{Proof. This is essentially a special case of Corollary 1.1.}

Two Quasi-Remarks. Beaumont and Lawver prove the theorem above for torsion-free strongly semi-simple groups, but obtain strong indecomposability as a conclusion. A torsion free group is quasidecomposable (the negation of strongly indecomposable) if $Q \otimes E$ contains an idempotent $e$, in which case $m e \in E$ for some nonzero integer $m$. The above proof goes through using me in place of $\alpha$.

Secondly, if $G$ is torsion free and $H$ is a subgroup such that $m G \leqq H \subseteq G$ for some integer $m \neq 0$ (i.e., $H \doteq G$ ), and if $\varphi$ is a ring on $H$, then $m^{2} \varphi$ is a ring on $G$. It is clear from this that full faithfulness is a quasi-isomorphism invariant.

Using one or the other of the above remarks we get

THEOREM 2. ([3]) If $G$ is a torsion free fully faithful abelian group then $G$ is strongly indecomposable.

We can get a weak converse to this result as follows. For a strongly indecomposable torsion free group of finite rank each element of $Q \otimes E$ is either nilpotent or a unit; and the radical, $N$, of $Q \otimes E$ is the set of nilpotent elements (cf. [5], Theorem 4.2, Corollary 4.3). Therefore, if $R$ is a nonnilpotent subring of $E$, then $R \nsubseteq N$ so for some $\rho \in R, \rho^{-1} \in Q \otimes E$. Then $m G \leqq \rho G \leqq G$ with $m \in Z$. Apply $\varphi$ to obtain $m R \subseteq \rho R \subseteq R$, so $m \rho=\rho \sigma$ for some $\sigma \in R$. Since $\rho$ is monic, $m=\sigma$. Thus $R$ contains a nonzero integer $m$. This remark is the basis of the proof of

THEOREM 3. If $G$ is torsion free of finite rank and strongly indecomposable then for every ring $\varphi$ on $G$, either $\varphi$ is faithful or $\varphi(G)$ is nilpotent.

Proof. Suppose $\varphi$ is a ring on $G$ and $R=\varphi(G)$ is not nilpotent. 
As above $R$ contains a nonzero integer $m$, and we denote by $K$ the kernel of $\varphi$. Choose $x \in G$ such that $\varphi(x)=m$ and define a map $\gamma: R \rightarrow G$ by $\gamma(\rho)=\rho x, \rho \in R$. Then $\varphi(\gamma(\rho))=\varphi(\rho x)=\rho \varphi(x)=m \rho$. This shows that $\gamma$ is $1-1$ and that $\gamma(R) \cap K=0$ so that $K+\gamma(R)=$ $K \oplus \gamma(R) \subseteq G$. Now for $g \in G, \quad \varphi(\gamma \varphi(g))=\varphi(\varphi(g) x)=\varphi(g) \varphi(x)=$ $m \varphi(g)=\varphi(m g)$ so that $\gamma \varphi(g)-m g \in K$. Thus $m g \leqq \gamma(R) \oplus K \subseteq G$ so $G \doteq K \oplus \gamma(R)$. Since $\gamma(R) \neq 0$ we must have $K=0$ as required.

We note that $\varphi(G)$ is nilpotent if and only if the ring $(G, \varphi)$ is nilpotent; perhaps this latter is a more natural hypothesis. We note also that the two alternative conclusions of the theorem can hold simultaneously for no group, for if $R=\varphi(G)$ is nilpotent, and $n$ is least such that $R^{n}=0$, choose $x \in G$ such that $\varphi(x) \in R^{n-1}, \varphi(x) \neq 0$. Then $\varphi(x) R=0$ so for all $y \in G$,

$$
0=\varphi(x) \varphi(y)=\varphi(\varphi(x) y) .
$$

If $\varphi$ were $1-1$, we would have $\varphi(x) y=0$ for all $y \in G$; i.e., $\varphi(x)=0$, contrary to the choice of $x$.

The proof of the theorem above suggests that of the following.

THEOREM 4. Suppose $\varphi$ is a ring on $G$ such that $(G, \varphi)$ has an identity. Then $\varphi$ is faithful and $\varphi(G)$ is a direct summand of $E$ (as abelian groups).

Proof. Let $x$ be the identity of $(G, \varphi)$. Then it is well known (and obvious) that the left regular representation of $(G, \varphi)$, namely $\varphi$, is $1-1$. Moreover, $\varphi(x)=1 \in E$. Put $R=\varphi(G)$ and define a $Z$-map $\nu: E \rightarrow R$ by $\nu(\varepsilon)=\varphi(\varepsilon x)$. Viewing $E$ as left $R$-module, equation (1) states that $\nu \in \operatorname{Hom}_{R}(E, R)$, and clearly $\nu$ is an epimorphism. Since $R$ is projective over itself, our conclusion follows.

In [5] we called a torsion free group irreducible provided it had no pure fully invariant subgroups (except 0 and itself). Let us call such a group $G$ strongly irreducible provided $G \doteq H$ whenever $H$ is a nonzero fully invariant subgroup of $G$. Then we can state the following theorem, which contains part of the main result of [3].

THEOREM 5. Let $G$ be a strongly indecomposable torsion free abelian group of finite rank. Then the following are equivalent:

(i) $G$ is semi-simple.

(ii) $G$ is strongly semi-simple.

(iii) $G$ is quasi-isomorphic to the integrally closed subring $J_{\pi}$ of an algebraic number field $K$ determined by an infinite (or empty) set $\pi$ of primes in $K$, and $[K: Q]=\operatorname{rank} G$. 
(When these conditions hold, $Q \otimes E=K, E \doteq J_{\pi}$ and the class of rings $(G, \varphi)$ on $G$ coincides with the quasi-equality class determined by $J_{\pi}$ in $K$. Moreover, $G$ is strongly irreducible.)

Proof. First let $G$ be semi-simple and let $\varphi$ be a semi-simple ring on $G$, with $R=\varphi(G)$. We know then (Corollary 0 ) that $\varphi$ is faithful. By the remarks preceding Theorem 3 and the fact that $R$ is semi-simple, so has no nilpotent ideals, $R$ consists of quasi-automorphisms (and 0 ) and $Q \otimes R$ is a division algebra. Since $\varphi$ is $1-1$ and $R$ is a full subring of $Q \otimes R$, it follows from [5], Theorem 5.4(i), that $G$ is irreducible and from [5], Theorem 5.5(iii), that $Q \otimes R=Q \otimes E$ so $Q \otimes E$ is a division algebra. Moreover, the latter reference gives $[Q \otimes E: Q]=\operatorname{rank} G$. Now $G$ being strongly indecomposable, the same is true of $R$, hence by [5], Corollary 5.7, $Q \otimes E$, and with it $E$, is commutative. Thus $K=Q \otimes E$ is an algebraic number field with $[K: Q]=\operatorname{rank} G$.

Now let $H$ be a nonzero subgroup of $G$ such that $R H \subseteq H$. Then equation (1) implies that $\varphi(H)$ is an ideal of $R$, nonzero, and hence contains some integer $m\left(\rho \in \varphi(H), \rho \neq 0\right.$ imply $m \rho^{-1} \in R$ for some nonzero $m$ so $\left.m \rho^{-1} \rho=m \in \varphi(H)\right)$. Thus $\varphi(H) \doteq R$, so $H \doteq G$. This has two consequences. In the first place $G$ is strongly irreducible since if $H$ is fully invariant then certainly $R H \subseteq H$. Secondly, if $x \in G$, $x \neq 0$ then $R x \doteq G$. Combining these two remarks we obtain $E x \doteq$ $G \doteq R x$. Clearly now $R \doteq E$.

The relation $R \doteq E$ or, say, $m E \subseteq R$, implies that $E$ is semisimple since $m E$, as an ideal of both $R$ and $E$ satisfies $(\operatorname{rad} E) \cap m E=$ $\operatorname{rad}(m E)=(\operatorname{rad} R) \cap m E=0, R$ being semi-simple. Clearly now any ring in the quasi-equality class determined by $E$ is semi-simple. In particular the integral closure, $J_{\pi}$, of $E$ is semi-simple, (that $E \doteq J_{\pi}$ is not hard to see; cf. also [2]), so $\pi$ is infinite (or empty-but then $J_{\pi}=K$ so $E=K$ and $G$ is not strongly indecomposable except in the trivial case $G=Q$, which we now ignore). We now have (iii).

Now let $\varphi^{\prime}$ be any ring on $G, \varphi^{\prime} \neq 0$. By Theorem $3, \varphi^{\prime}$ is faithful since $E$ contains no nilpotent elements. Thus $R^{\prime}=\varphi^{\prime}(G)$ is a full subring of $E$. The map $\varphi(x) \rightarrow \varphi^{\prime}(x), x \in G$ is clearly a well defined additive map of $R$ onto $R^{\prime}$ and, viewing $R, R^{\prime}$ as modules over $R \cap R^{\prime}$, this map is a module map. Since $R \cap R^{\prime}$ is full in $K$, this map extends to a $K$-linear map of $K$ onto $K$, hence is given by multiplication by some element of $K$. Thus $R^{\prime}=\alpha R$ for some $\alpha \in K$. Since $R$ and $R^{\prime}$ are full in $K$ there is a nonzero integer $n$ such that $n \alpha^{-1} \in R \cap R^{\prime}$. Then $R^{\prime} \supseteqq n \alpha^{-1} R^{\prime}=n \alpha^{-1}(\alpha R)=n R$. Similarly $n R^{\prime} \subseteq R$ 
so $R \doteq R^{\prime}$. Thus too $R^{\prime} \doteq E$, so is semi-simple and (ii) holds. We have evidently established the last statement of the theorem as well.

We have now shown that (i) implies all other statements in the theorem. Clearly (ii) implies (i) as does (iii) in view of the fact that the $J_{\pi}$ in (iii) is semi-simple and semi-simplicity is a quasi-isomorphism invariant. (This follows from the second quasi-remark and the argument given above showing that $R$ semi-simple and $R \doteq E$ implies $E$ semi-simple. Beaumont-Lawver give an explicit proof as well.)

Our discussion so far has been based solely on fairly general facts drawn from [5]. Using the Beaumont-Pierce analogue of the Wedderburn Principal Theorem [1] we can obtain our final results. The Beaumont-Pierce Theorem states that any torsion free ring is quasi-equal to the group direct sum of its maximal nilpotent ideal $N$ and a subring $S$.

In the following theorem we give an alternative proof of the part of the main results of [3] not included in Theorem 5. A group is anti-radical if $(G, \varphi)$ is never a radical ring for all rings $\varphi \neq 0$ on $G$.

THEOREM 6. A strongly indecomposable torsion free group of finite rank is semi-simple if and only if it is nonnil and antiradical.

Proof. A semi-simple group is trivially nonnil and anti-radical. Conversely let $\varphi$ be a ring on $G$ such that $(G, \varphi)$ is not a radical ring. Then $\varphi(G)$ is not nilpotent so by Theorem 3, $\varphi$ is faithful and $G \cong \varphi(G)=R$ as groups. By the Beaumont-Pierce Theorem, strong indecomposability of $\mathrm{G}$ and the fact that $R$ is not nilpotent, the maximal nilpotent ideal of $R$ is zero. By arguments used before (e.g. Theorem 3, or [5] Theorem 4.2, Corollary 4.3) $R$ consists of quasi-automorphisms of $G$. By other arguments used before (Theorem 5) any nonzero ideal of $R$ is quasi-equal to $R$. If $\operatorname{rad} R \neq 0$ then $m R \subseteq \operatorname{rad} R \subseteq R$ for some integer $m \neq 0$. This implies as in the proof of Theorem 5 that $\operatorname{rad} m R=m R$. But then $m \varphi$ is a nonzero radical ring on $G$.

As another application of the Beaumont-Pierce Theorem we strengthen Theorem 3 in

THEOREM 7. Let $G$ be a strongly indecomposable faithful group of finite rank. Then $G$ is strongly irreducible, fully faithful and its 
endomorphism ring $E$ is a subring of an algebraic number field whose degree is the rank of $G$. Moreover, the class of rings on $G$ coincides with the quasi-equality class of $E$.

Proof. Let $\varphi$ be a faithful ring on $G$ and put $R=\varphi(G)$. Then $R$ is strongly indecomposable as a group and by the remark following Theorem $3, R$ is not nilpotent so by the Beaumont-Pierce Theorem the maximal nilpotent ideal of $R$ is zero. By familiar arguments we conclude that $R$ consists of quasi-automorphisms of $G$ and that therefore every nonzero ideal (left, right or two-sided) of $R$ is quasiequal to $R$. Clearly $R$ is strongly irreducible as a group, so $G$ is too. But then ([5], Theorem 5.5(iii)) $Q \otimes E$ is a division algebra with $[Q \otimes E: Q]=\operatorname{rank} G$, and in fact ([5], Corollary 5.7) commutative. As in the proof of Theorem $5, R \doteq E$. Finally, since $E$ has no nilpotent elements, Theorem 3 implies that $G$ is fully faithful.

Corollary 7.1. Let $G$ be a strongly indecomposable group of finite rank. Then exactly one of the following hold:

(i) $G$ is fully faithful.

(ii) $G$ is nil.

(iii) $G$ is nonnil and for every ring $\varphi$ on $G,(G, \varphi)$ is nilpotent. Moreover, the first alternative holds if and only if $G$ is strongly irreducible. If $G$ is irreducible but not strongly irreducible, then $G$ is nil.

Proof. Theorems 3 and 7 show that exactly one of the three alternatives hold; and that if (i) holds then $G$ is strongly irreducible. Conversely if $G$ is strongly indecomposable and strongly irreducible then $Q \otimes E$ is a division algebra of dimension equal to the rank of $G$. If $x \in G, x \neq 0$ then the map $\varepsilon \rightarrow \varepsilon x, \varepsilon \in E$, is a monomorphism of $E$ into $G$ whose image is fully invariant, hence quasi-equal to $G$. Thus $G$ is quasi-isomorphic to $E$, and $E$ is patently faithful, so $G$ is faithful too. Finally, if $G$ is irreducible but not strongly irreducible, then (iii) cannot hold as $Q \otimes E$ is a division algebra and (i) cannot hold, so (ii) alone remains.

These last results make it convenient for us to exhibit a class of fully faithful groups which are not strongly semi-simple, thus indicating that various of our results are indeed generalizations of some of those in [3]. We start with an algebraic number field $F$ and choose a rational prime $p$ such that $F$ is contained in the field of $p$-adic numbers. The condition for this is that $p$ be unramified and of degree 1 in $F$, and it is well known that such primes $p$ exist. Let $P$ be the ring of $p$-adic integers and put $R=F \cap P$. Then $R$ 
is not semi-simple and, as an additive group, $R$ is pure in $P$ so is strongly indecomposable. By Corollary 7.1 the additive group of $R$ is fully faithful.

COROLlaRy 7.2 [6]. If $G$ is strongly indecomposable and admits one nilpotent nonzero multiplication, then $G$ is radical (i.e., every ring on $G$ is nilpotent).

It is also shown in [6] that a torsion free group of finite rank is radical if and only if $Q \otimes E(A)$ is never a field, for $A$ a quasisummand of $G$. In our case ( $G$ strongly indecomposable) this simply means that $Q \otimes E$ is not a field. This result too follows from Corollary 7.1. In closing we note that Fuchs [4, p. 311] has asked for a survey of the rings on strongly indecomposable torsion free groups of finite rank. Our results at least give such a survey in case (i) above.

\section{REFERENCES}

1. R. A. Beaumont and R. S. Pierce, Torsion free rings, Illinois J. Math., 5 (1961), 61-98.

2. - Subrings of algebraic number fields, Acta Sci. Math. Szeged, 22 (1961), 202-216.

3. R. A. Beaumont and D. A. Lawver, Strongly semi-simple abelian groups, Pacific J. Math., 48 (to appear).

4. L. Fuchs, Infinite Abelian Groups, v. II, Academic Press, New York, 1973.

5. J. D. Reid, On the Ring of Quasi-Endomorphisms of a Torsion Free Group, Topics in Abelian Groups, Scott Foresman and Company, Chicago, 1963.

6. W. J. Wickless, Abelian groups which admit only nilpotent multiplications, Pacific J. Math., 40 (1972), 251-259.

Received July 1, 1973 and in revised form September 17, 1973. This work was supported in part by the National Science Foundation.

WESLEYAN UNIVERSITY 



\section{PACIFIC JOURNAL OF MATHEMATICS}

\section{EDITORS}

RICHARD ARENS (Managing Editor)

University of California

Los Angeles, California 90024

R. A. Beaumont

University of Washington

Seattle, Washington 98105
J. DugundJI

Department of Mathematics

University of Southern California

Los Angeles, California 90007

D. Gilbarg and J. Milgram

Stanford University

Stanford, California 94305

\section{ASSOCIATE EDITORS}

E. F. BECKENBACH

B. H. NeUMANN

F. WOLF

K. YosHIDA

\section{SUPPORTING INSTITUTIONS}

UNIVERSITY OF BRITISH COLUMBIA

CALIFORNIA INSTITUTE OF TECHNOLOGY

UNIVERSITY OF CALIFORNIA

MONTANA STATE UNIVERSITY

UNIVERSITY OF NEVADA

NEW MEXICO STATE UNIVERSITY

OREGON STATE UNIVERSITY

UNIVERSITY OF OREGON

OSAKA UNIVERSITY
UNIVERSITY OF SOUTHERN CALIFORNIA

STANFORD UNIVERSITY

UNIVERSITY OF TOKYO

UNIVERSITY OF UTAH

WASHINGTON STATE UNIVERSITY

UNIVERSITY OF WASHINGTON

$\stackrel{*}{*} \stackrel{*}{*} \stackrel{*}{*}$ AMERICAN MATHEMATICAL SOCIETY




\section{Pacific Journal of Mathematics}

\section{Vol. 53, No. $1 \quad$ March, 1974}

Martin Bartelt, Strongly unique best approximates to a function on a set, and a finite

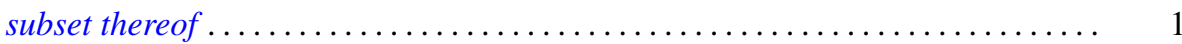

S. J. Bernau, Theorems of Korovkin type for $L_{p}$-spaces $\ldots \ldots \ldots \ldots \ldots \ldots \ldots \ldots \ldots$

S. J. Bernau and Howard E. Lacey, The range of a contractive projection on an

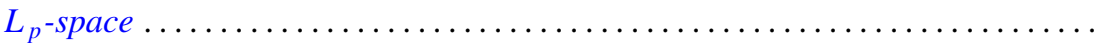

Marilyn Breen, Decomposition theorems for 3-convex subsets of the plane ......... Ronald Elroy Bruck, Jr., A common fixed point theorem for a commuting family of

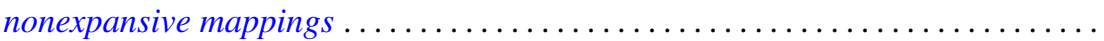

Aiden A. Bruen and J. C. Fisher, Blocking sets and complete $k$-arcs . . . . . . . 73

R. Creighton Buck, Approximation properties of vector valued functions . ......... 85

Mary Rodriguez Embry and Marvin Rosenblum, Spectra, tensor products, and

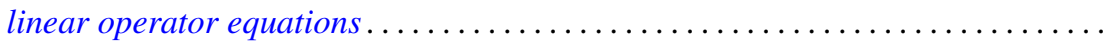

Edward William Formanek, Maximal quotient rings of group rings . . . . . . . . . 109

Barry J. Gardner, Some aspects of T-nilpotence . . . . . . . . . . . . . . . 117

Juan A. Gatica and William A. Kirk, A fixed point theorem for $k$-set-contractions

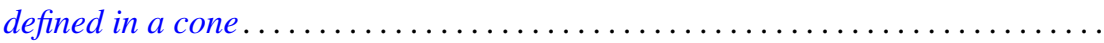

Kenneth R. Goodearl, Localization and splitting in hereditary noetherian prime

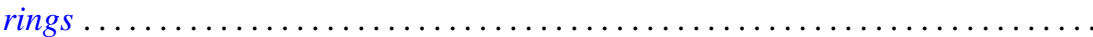

James Victor Herod, Generators for evolution systems with quasi continuous

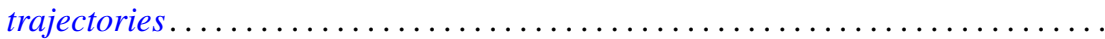

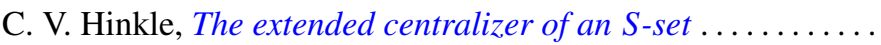

I. Martin (Irving) Isaacs, Lifting Brauer characters of p-solvable groups . . .

Bruce R. Johnson, Generalized Lerch zeta function ...........

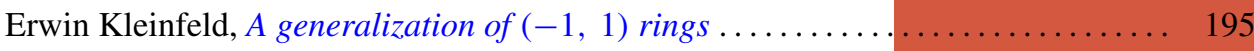

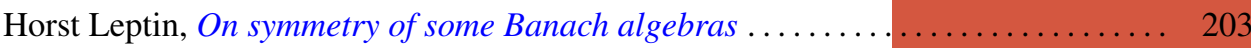

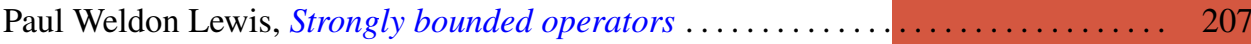

Arthur Larry Lieberman, Spectral distribution of the sum of self-adjoint

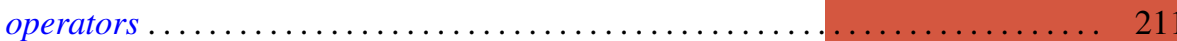

I. J. Maddox and Michael A. L. Willey, Continuous operators on paranormed spaces and matrix transformations

James Dolan Reid, On rings on groups ........................... 229

Richard Miles Schori and James Edward West, Hyperspaces of graphs are Hilbert cubes.

William H. Specht, A factorization theorem for p-constrained groups ...

Robert L Thele, Iterative techniques for approximation of fixed points of certain nonlinear mappings in Banach spaces ...............

Tim Eden Traynor, An elementary proof of the lifting theorem

Charles Irvin Vinsonhaler and William Jennings Wickless, Completely decomposable groups which admit only nilpotent multiplications .

Raymond O’Neil Wells, Jr, Comparison of de Rham and Dolbeault cohomology for

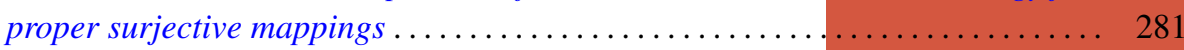

David Lee Wright, The non-minimality of induced central representations . . . . . 301 